\title{
Sialic Acid on the Neuronal Glycocalyx Prevents Complement C1 Binding and Complement Receptor-3-Mediated Removal by Microglia
}

\author{
Bettina Linnartz, ${ }^{1}$ Jens Kopatz, ${ }^{1}$ Andrea J. Tenner, ${ }^{2}$ and Harald Neumann ${ }^{1}$ \\ ${ }^{1}$ Neural Regeneration Group, Institute of Reconstructive Neurobiology, University Hospital Bonn, 53127 Bonn, Germany, and ${ }^{2}$ Department of Molecular \\ Biology and Biochemistry, Institute for Immunology, University of California, Irvine, California 92697
}

Microglial cells are professional phagocytes of the CNS responsible for clearance of unwanted structures. Neuronal processes are marked by complement $\mathrm{C} 1$ before they are removed in development or during disease processes. Target molecules involved in $\mathrm{C} 1$ binding and mechanisms of clearance are still unclear. Here we show that the terminal sugar residue sialic acid of the mouse neuronal glycocalyx determines complement $\mathrm{C} 1$ binding and microglial-mediated clearance function. Several early components of the classical complement cascade including $\mathrm{C1q}, \mathrm{C1r}, \mathrm{C} 1 \mathrm{~s}$, and $\mathrm{C} 3$ were produced by cultured mouse microglia. The opsonin $\mathrm{Clq}$ was binding to neurites after enzymatic removal of sialic acid residues from the neuronal glycocalyx. Desialylated neurites, but not neurites with intact sialic acid caps, were cleared and taken up by cocultured microglial cells. The removal of the desialylated neurites was mediated via the complement receptor-3 (CR3; CD11b/CD18). Data demonstrate that mouse microglial cells via CR3 recognize and remove neuronal structures with an altered neuronal glycocalyx lacking terminal sialic acid.

\section{Introduction}

Microglial cells originate from myeloid cells of the hematopoietic lineage and populate the CNS during early neuroectodermal development (Ransohoff and Cardona, 2010). Silent phagocytosis of apoptotic cells without inflammation is one of the major beneficial functions of microglia (Neumann et al., 2009). The cellular machinery for phagocytosis of apoptotic bodies appears to be conserved across species and involves an immunoreceptor tyrosine-based activation motif (ITAM) (Ziegenfuss et al., 2008). In mammals ITAM-signaling in microglia is at least partially mediated via DAP12 or Fc-receptors. Recently, it has been shown in vitro that triggering receptor expressed on myeloid cells-2 (TREM2)-mediated signaling via DAP12 in mouse microglia facilitates clearance of apoptotic cells in the absence of inflammation (Takahashi et al., 2005). The complement receptor 3 (CR3), a major heterodimeric microglial receptor consisting of the integrins CD11b and CD18, also signals via DAP12 (Ivashkiv,

Received July 27, 2011; revised Nov. 16, 2011; accepted Nov. 25, 2011.

Author contributions: B.L. and H.N. designed research; B.L. and J.K. performed research; A.J.T. contributed unpublished reagents/analytic tools; B.L. and J.K. analyzed data; B.L., A.J.T., and H.N. wrote the paper.

This project was supported by the Deutsche Forschungsgemeinschaft (KF0177, SFB704, FOR1336), the Hertie Foundation, and the University of California, Irvine contribution by NIH AG 00538. The Neural Regeneration Group at the University Bonn LIFE \& BRAIN Center was supported by the Hertie Foundation and Walter-und-Ilse-RoseFoundation. All animal experiments were approved by the authors' institutional review boards and by the local government and were conducted according to the principles expressed in the Helsinki Declaration. We thank Ajit Varki for helpful discussion. We thank Rita Hass and Jessica Schumacher for excellent technical support of cultures and molecular biology.

The authors declare no competing financial interests.

Correspondence should be addressed to Harald Neumann, Institute of Reconstructive Neurobiology, University Bonn, Sigmund-Freud-Strasse 25, 53127 Bonn, Germany. E-mail: hneuman1@uni-bonn.de.

DOI:10.1523/JNEUROSCI.3830-11.2012

Copyright $\odot 2012$ the authors $\quad 0270-6474 / 12 / 320946-07 \$ 15.00 / 0$
2009). Evidence for an involvement of CR3 and DAP12 in the clearance of neurons during development came from a study by Wakselman et al. (2008). They showed that apoptotic neurons in the developing hippocampus are contacted and cleared by CR3 of microglia. Recent data indicate that microglia are involved in synaptic pruning during postnatal development in mice (Paolicelli et al., 2011). Moreover, during normal brain development or before degeneration of adult retinal neurons synapses and axons are marked by the complement components $\mathrm{Clq}$ and C3 before they are removed (Stevens et al., 2007). However, it remained unclear which target structures are recognized by the complement components and which cell type and receptors are involved in the removal of the neuronal structures.

C1q can bind several target structures including bacteria, Igs, and pentraxins (Inforzato et al., 2006; Lund et al., 1996; Khatua et al., 2010). The binding of C1q on the target structures can depend on the terminal sugar residues (Inforzato et al., 2006; Lund et al., 1996). Upon initiation of the classical complement pathway via binding of $\mathrm{C} 1 \mathrm{q}$ to target structures, $\mathrm{C} 2$ and $\mathrm{C} 4$ are activated by cleavage leading to the cleavage of $\mathrm{C} 3$ and covalent opsonization by $\mathrm{C} 3$ fragments on the surface of target cells followed by recognition via CR3 and the removal by phagocytes (Fraser and Tenner, 2008). In the present study we show that the enzymatic desialylation of the neuronal glycocalyx allowed complement C1q binding and CR3mediated removal of neurites by microglia.

\section{Materials and Methods}

Neuronal and microglial culture. Primary neuronal cultures were obtained from embryonic mouse C57BL/6 hippocampal tissue of either sex (Gorlovoy et al., 2009) and primary microglial cultures from brains of postnatal day 3 or 4 C57BL/6 mice of either sex (Napoli et al., 2009). Embryonic stem cell-derived microglial cells (ESdMs) that were shown 
to be very similar to primary cultured microglia were used in all other experiments involving microglia (Takahashi et al., 2005; Beutner et al., 2010). Unstimulated microglia (ESdMs) are capable of chemokinedirected migration and phagocytosis, the latter being increased following proinflammatory stimulation (Beutner et al., 2010). For coculture experiments microglia (ESdMs) were lentivirally transduced with green fluorescent protein (GFP-ESdMs) as described previously (Takahashi et al., 2005; Beutner et al., 2010).

Immunocytochemistry of complement C1q and C3. Primary neurons were either untreated or treated with $12.5 \mathrm{U} / \mathrm{ml}$ exo- $\alpha$-sialidase (neuraminidase, EC 3.2.1.18, BioLabs) plus $0.5 \mathrm{U} / \mathrm{ml}$ endo- $\alpha$-sialidase (endoneuramidase $\mathrm{N}, \mathrm{EC} 3.2 .1 .129$, Abcys) for $2.5 \mathrm{~h}$ at $37^{\circ} \mathrm{C}$ to remove $\alpha 2.3-, \alpha 2.6-$, and $\alpha 2.8$-linked sialic acid residues. Cells were incubated for $1 \mathrm{~h}$ at $37^{\circ} \mathrm{C}$ in mouse or human serum, human purified C1q (Benoit and Tenner, 2011) or human C1-depleted serum (Benoit and Tenner, 2011). Cells were fixed and stained with rat monoclonal anti-mouse $\mathrm{Clq}$ (1:200, Abcam), murine monoclonal anti-human C1q (1:200, Quidel) or rat monoclonal anti-mouse $\mathrm{C} 3 \mathrm{~b} / \mathrm{C} 3 \mathrm{bi} / \mathrm{C} 3 \mathrm{c}$ ( $1: 50$, Hycult) followed by the corresponding Cy3-conjugated secondary antibodies (1:200, Jackson ImmunoResearch Laboratories). Cells were double labeled with antiIba-1 (ionized calcium binding adaptor molecule 1, 1:2000, Wako) for microglia or anti- $\beta$ III-tubulin (1:500, Sigma-Aldrich) for neurons, followed by FITC-conjugated secondary antibodies (1:200). Nuclei of cells were subsequently labeled with 4',6-diamidino-2-phenylindole (DAPI, 1:5000, Sigma-Aldrich). Images were taken by confocal laser scanning microscopy (Fluoview 1000, Olympus). For quantification of C1q staining intensity, in total 15 pictures per condition out of 3 independent experiments were collected maintaining the same settings and analyzed by ImageJ software (NIH) by a blinded investigator. After subtracting the background, the measured mean values of the staining intensities were compared.

Reverse transcriptase PCR analysis of complement genes. Total RNA of cells was isolated using the RNeasy Mini Kit (Qiagen) for analysis of C1q, $\mathrm{C} 1 \mathrm{r}, \mathrm{C} 1 \mathrm{~s}$, and $\mathrm{C} 3$ gene transcription. Cells were either untreated or treated for $24 \mathrm{~h}$ with the following substances: $500 \mathrm{ng} / \mathrm{ml}$ LPS (SigmaAldrich), $100 \mathrm{U} / \mathrm{ml}$ mouse IFN $\gamma$ (R\&D Systems), or $1000 \mathrm{U} / \mathrm{ml}$ mouse IFN $\alpha$ (Hycult Biotech). Reverse transcription was performed and the resulting cDNA was amplified by PCR. Quantitative real-time reverse transcriptase (RT)-PCR was performed with SYBR Green PCR Master Mix (Qiagen) using the ABI 5700 Sequence Detection System (PerkinElmer). The $\Delta \Delta C_{\mathrm{T}}$ method with GAPDH as internal standard was used. The following oligonucleotides were used for RT-PCR and real-time PCR: GAPDH, forward (F): ACAACTTTGGCATTGTGGAA, reverse (R): GATGCAGGGATGATGTTCTG; C1q (RT-PCR), F: CCAGTATGTGGGCAGACCTC, R: TGAAGCCAGAGAAAGTGCTG; C1q (realtime PCR), F: CTGCAAGAACGGCCAGGTGC, R: AGCCACGGATGA AAAGGGGTG; C1r, F: CGTGCCAGTCTGTGGAAGACCA, R: TGGG CAGCCGTCAGGATCCA; C1s, F: AGGAAGAGGGAAAGACAAGGAA CCA, R: CCAAACACCACATCCCTGGCGA, C3 (RT-PCR), F: GGGG ACAACCTCAATGTCAA，R: CCAGGCGAAATGAAGGAATA; C3 (real-time PCR), F: TAGTGCTACTGCTGCTGTTGGC, R: GCTGGAATCTTGATGGAGACGCTT.

Flow cytometry of CD11b and CD18. Cells were mechanically detached, incubated for Fc-receptor blockade with anti-CD16/CD32 antibody (1: 100, Fc-block, BD Biosciences), and stained with a biotin-conjugated anti-CD11b or anti-CD18 antibody (1:100, BD Biosciences), followed by phycoerythrin-conjugated streptavidin (1:200, BD Biosciences). Isotypematched control antibodies (1:100, BD Bioscience) were used as negative controls. Analysis was done with a FACSCalibur flow cytometer (BD Biosciences) and analysis was performed with FlowJo Software.

Neurite length analysis after coculture with microglia and phagocytic uptake of neurites into microglia. Primary neurons were either untreated or treated with both sialidases (see Immunocytochemistry of complement $\mathrm{C} 1 \mathrm{q}$ and $\mathrm{C} 3$, above). Microglia $\left(5 \times 10^{4}\right)$ and a blocking antibody directed against CD11b (2 $\mu \mathrm{g} / \mathrm{ml}$, PharMingen $)$ or rat $\operatorname{IgG}_{2} \mathrm{~b}, \kappa$ isotype control $(2 \mu \mathrm{g} / \mathrm{ml}$, PharMingen $)$ were added. After $24 \mathrm{~h}$ cells were fixed and immunostained with rabbit anti-Iba-1 (1:2000, Wako) followed by Alexa Fluor 488-conjugated secondary antibodies (1:500). Cells were double labeled with anti- $\beta$ III-tubulin followed by Cy3-conjugated secondary antibody (1:500).

For phagocytosis, primary neurons were incubated with the red membrane fluorescent dye PKH26 (10 nM final concentration, SigmaAldrich) for $5 \mathrm{~min}$ at $37^{\circ} \mathrm{C}$. GFP-transduced microglia $\left(5 \times 10^{4}\right)$ were added for $24 \mathrm{~h}$. As a positive control, $2500 \mu \mathrm{g}$ of prestained (celltracker cM-DiI, Invitrogen; according to supplier's manual) apoptotic material was added to the coculture system. Apoptotic material was generated by cultivation without medium change for several days until cells underwent apoptosis. Cells were fixed and nuclei were labeled with DAPI. Images (normal or $z$-stack, 5-10 randomly selected areas in each group) were collected with a laser scanning confocal microscope (Fluoview 1000, Olympus) or fluorescent microscope (Axioskop2, Zeiss) by a blinded observer. Images were equally processed and the mean length of $\beta$ III-tubulin-positive neurites or the uptake of red fluorescent labeled material was quantified by ImageJ/NeuronJ software (NIH).

Statistical analysis. Data are presented as mean \pm SEM of at least three independent experiments. Data were analyzed by Student's $t$ test $(2$ groups only) or ANOVA ( $>2$ groups) followed by Bonferroni using SPSS computer software.

\section{Results \\ Binding of complement Clq to the desialylated neuronal glycocalyx}

Cultured neurons were treated with a combination of an exo- and endo- $\alpha$-sialidase to remove all sialic acid residues on the cell surface. Absence of sialic acids was confirmed with a soluble sialic acid-binding immunoglobulin superfamily lectin-F (SiglecF)-Fc fusion protein, which recognizes $\alpha 2.3$ sialic acid with preference for $6^{\prime}$-sulfo-sialyl Lewis X (Tateno et al., 2005). In the untreated control situation Siglec-F-Fc immunostained sialic acids can be observed on the normal neuronal glycocalyx, while a strong reduction in Siglec-F-Fc immunostaining was detected after challenging the neurons with both sialidases (data not shown). Neuronal cell number and neurite density were unaffected by the enzymatic treatment. In detail, analysis of neurons at $24 \mathrm{~h}$ after enzymatic treatment showed no significant change in relative neuronal cell body density $(96.78 \pm 3.22)$ and neurite length $(87.83 \pm 8.04)$ compared with the untreated controls $100 \pm 4.5$ and $100 \pm 5.94$, respectively. To investigate whether desialylation of the neuronal glycocalyx might initiate complement binding, neurons were treated with sialidases and then incubated with purified human C1q or complement containing human or mouse serum. Neurons were then analyzed by immunocytochemistry with specific antibodies directed against human or mouse C1q. Analysis of neurons incubated with purified human $\mathrm{C} 1 \mathrm{q}$ revealed increased binding of $\mathrm{Clq}$ to desialylated neurites (Fig. 1 $A, B$ ). Quantification of the bound and stained human purified $\mathrm{Clq}$ on neurons showed an increased binding of human C1q after sialidase treatment compared with the untreated situation (Fig. 1C). Binding of $\mathrm{C1q}$ was also detected on desialylated neurons and neurites after incubation of cultured neurons with human serum (Fig. 1C). As a control, neurons were incubated with C1q-depleted (C1qD) serum. The C1q staining of sialidasetreated neurons was increased after incubation in normal human serum compared with untreated neurons, while untreated or sialidase-treated neurons incubated in $\mathrm{C} 1 \mathrm{qD}$ serum showed almost no C1q staining (Fig. 1C). Neurons treated with sialidases also bound mouse C1q. After incubation with mouse serum sialidase-treated neurons showed increased ( $p=0.021)$ immunostaining with antibodies directed against $\mathrm{C1q}$ compared with neurons with an intact glycocalyx (data not shown). 
Components of the classical

complement cascade are produced by microglia

RT-PCR and immunocytochemistry were performed to analyze gene transcription and protein expression of complement components in neurons and microglia. In neuronal cultures containing few astrocytes no transcripts of complement components were detected by normal RTPCR (Fig. 2A), and only very low levels of $\mathrm{C} 1 \mathrm{q}$ transcripts were observed by realtime RT-PCR (data not shown). C1q, C1r, $\mathrm{C} 1 \mathrm{~s}$, and $\mathrm{C} 3$ were detected in microglia (Fig. 2A). To investigate whether the transcription of complement components is altered after different inflammatory stimuli, microglia were treated with LPS, IFN $\alpha$, or IFN $\gamma$. Treatment with IFN $\alpha$ did not change the transcription of $\mathrm{C} 1 \mathrm{q}$ or $\mathrm{C} 3$ (Fig. $2 \mathrm{~B}$ ). While stimulation with LPS had no effect on $\mathrm{Clq}$, gene transcription of $\mathrm{C} 3$ was increased (Fig. $2 \mathrm{~B}$ ). After stimulation with IFN $\gamma$ transcription of $\mathrm{Clq}$ was downregulated, while C3 was upregulated (Fig. 2B). Moreover, cultured microglia and neurons were double-immunostained with antibodies directed against $\mathrm{C} 1 \mathrm{q}$ or C3. While expression of C1q and C3 was detected in cultured microglial cells (Fig. 2C), cultured neurons showed no immunostaining for C1q or C3 (Fig. $2 D)$. Next, we stained microglia for CD11b and CD18 and analyzed them by flow cytometry. Both components were expressed at similar levels (Fig. 2E).

Involvement of complement receptor-3 in microglial removal of desialylated neurites

Neurons were either untreated or treated with both sialidases and then cocultured with microglia. Neurons and microglia were immunostained with antibodies directed against $\beta$ III-tubulin and Iba-1, respectively. Irrespectively of being cocultured with normal or desialylated neurons, the morphology of microglia remained unaltered (data not shown). Microglia without inflammatory stimulation did not affect neurite morphology, neurite length or neuronal survival (Fig. $3 A, C$ ). However, after removal of sialic acid on the neuronal cell surface, neurons cocultured with microglia showed a loss of neurites (Fig. $3 A, B$ ). The relative neurite length in neuron-microglia cocultures was reduced from $90.07 \pm 5.53 \%$ to $50.76 \pm$ $4.92 \%$ after removal of sialic acid from the neurons (Fig. 3C). The sialidase treatment alone did not change the relative neurite length (Fig. 3C).

Normal or desialylated neurons were cocultured with microglia and CR3 was blocked by an anti-CD11b antibody. The reduction in neurite length after coculture of microglia with

C
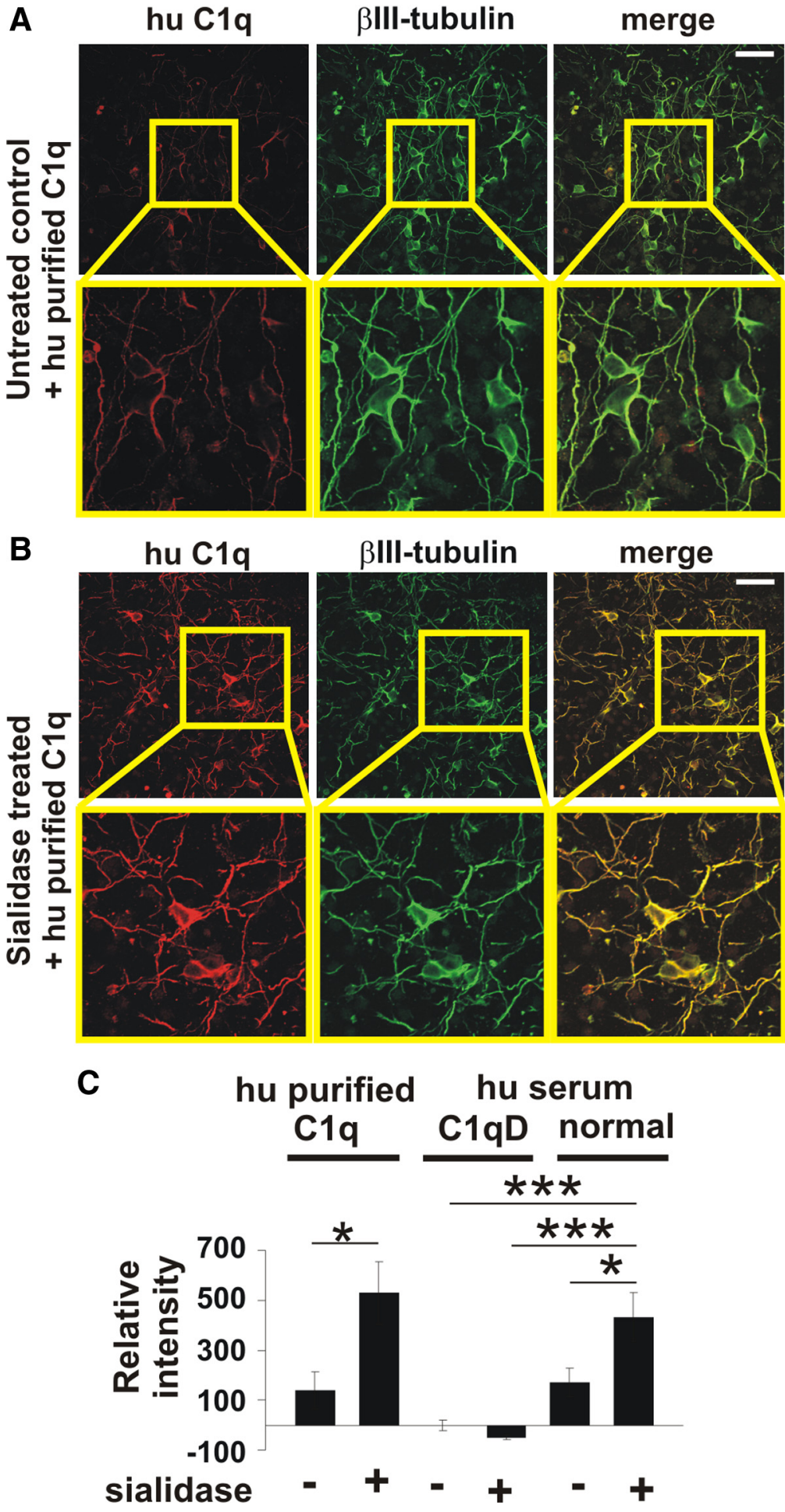

Figure 1. Binding of complement $\mathrm{C} 1$ to the desialylated neuronal glycocalyx. Cultured neurons were incubated with human purified C1q (hu purified (1q), serum containing complement (normal hu serum) or serum depleted for $\mathrm{C} 1 \mathrm{q}$ (hu serum C1qD). Cells were immunostained with an anti-C1q antibody and double-immunolabeled with $\beta$ III-tubulin. $A, B$, Confocal images of untreated or sialidase-treated neurons. Removal of sialic acids from the glycocalyxled to an increased C1q staining. Scale bar, $50 \mu \mathrm{m}$; higher magnification $115 \mu \mathrm{m}, n=$ 3. C, Quantification of $\mathrm{C}$ binding to neurons. Purified $\mathrm{C} 1 \mathrm{q}$ binding to desialylated neurons was increased compared with untreated neurons $\left({ }^{*} p=0.014\right)$. Binding of $C 1$ on desialylated neurons was also increased compared with untreated neurons $\left({ }^{*} p=0.013\right)$, untreated $\left({ }^{* * *} p=1.11 \times 10^{-5}\right)$ or sialidase-treated $\left.{ }^{* * *} p=1.14 \times 10^{-6}\right)$ neurons incubated in $C 1 q D$ serum. $n=3$.

desialylated neurons was reversed by addition of the CD11b blocking antibody $(87.79 \pm 9.30 \%)$, while control antibodytreated microglia also led to a reduction in neurite length (50.81 $\pm 2.64 \%$; Fig. $3 D)$. 

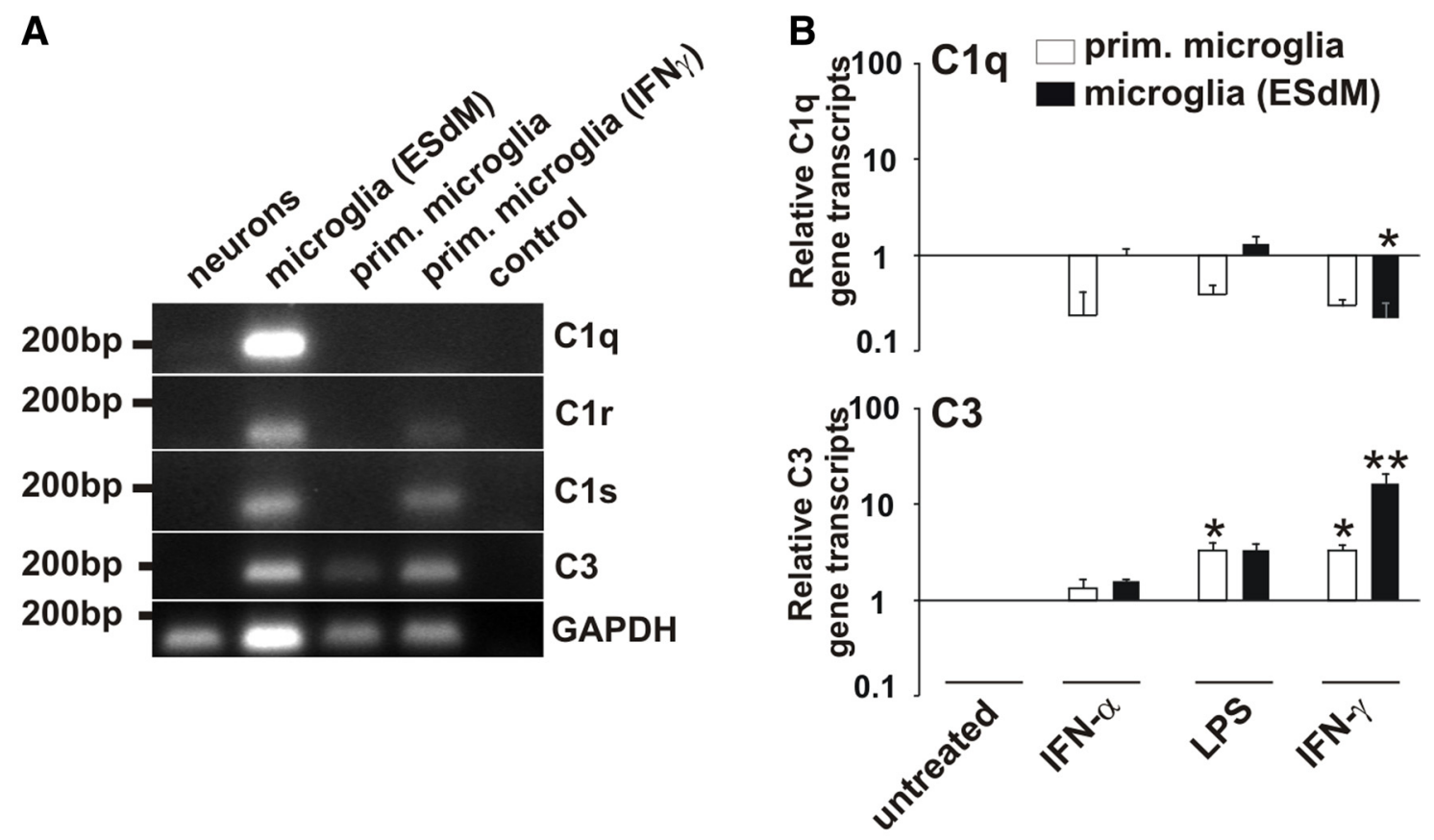

C

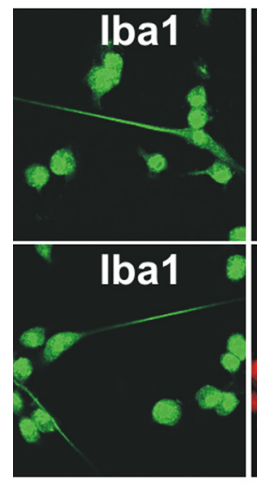

microglia

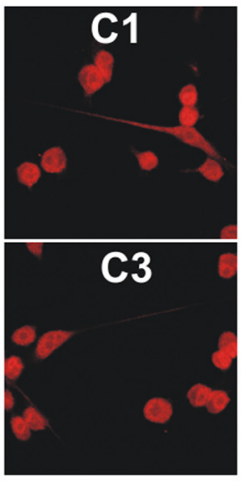

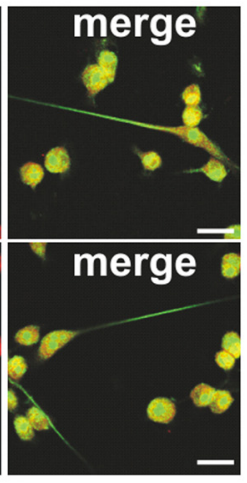

D

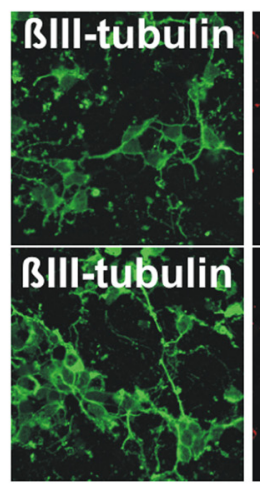

neurons
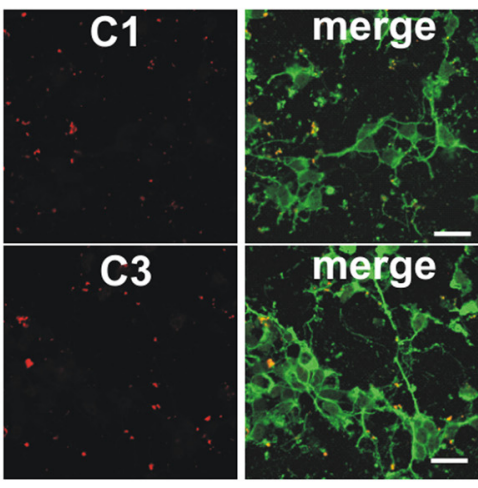

E microglia (ESdM)
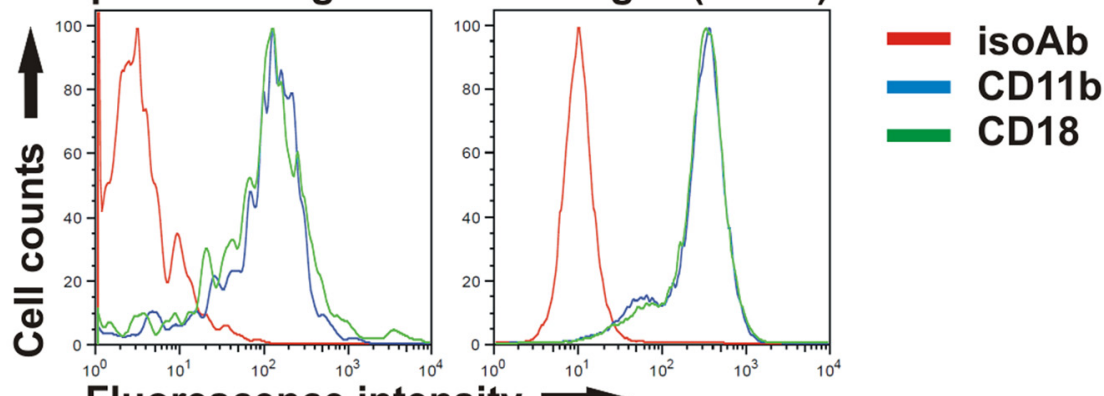

Figure 2. Complement production by microglia. $A$, RT-PCR of neurons, microglia (ESdMs) and primary (prim.) microglia. Transcripts for C1q, C1r, C1s, and C3 were detected in unstimulated microglia (ESdMs). C 3 was constitutively transcribed in primary microglia, while C1r and C1s were detected after IFN $\gamma$ stimulation. GAPDH served as standard. Control:PCR without cDNA. $n=3 . \boldsymbol{B}$, Microglia (prim. and ESdMs) were untreated or stimulated for $24 \mathrm{~h}$ with IFN $\alpha(1000 \mathrm{U} / \mathrm{ml})$, LPS (500 ng/ml), or IFN $\gamma(100 \mathrm{U} / \mathrm{ml})$. After LPS treatment transcripts of C 3 were upregulated (prim. microglia: $p=0.015 \mathrm{vs} \mathrm{untreated).} \mathrm{After} \mathrm{IFN} \gamma$ stimulation transcripts of (1q were downregulated (ESdMs: $p=0.024$ vs untreated), while (3 was upregulated (prim. microglia: $p=0.015$ vs untreated; ESdMs: $p=0.001$ vs untreated). $n=3$ for prim. microglia (C1q), $n=5$ for prim. microglia (C3), $n=4$ for ESdMs. C, D, Microglia and neurons were double-immunostained with antibodies directed against C $1 \mathrm{q} /(\mathrm{C} 3$ and Iba- $1 / \beta \mathrm{lll}$-tubulin. Protein expression of C1q and C3 was detected in microglia while it was undetectable in neurons. Scale bar, $20 \mu \mathrm{m}, n=3$.E, Flow cytometry of microglia (prim. and ESdMs). Expression of CD11b (blue) and CD18 (green) was detected at similar levels by prim. microglia and microglia (ESdMs). An isotype antibody (red) was used as negative control. $n=3$.

Microglial uptake of desialylated neuronal membranes To analyze whether microglia take up desialylated neuronal material, GFP-transduced microglia were either cocultured with normal or desialylated neurons, labeled with the red fluorescent membrane dye PKH26. The uptake of neuronal membranes into the microglia was visualized by confocal microscopy and 3Dreconstruction. Fluorescent-marked neuronal membranes were detected in the microglia after coculture with desialylated neu- 
rons (Fig. 4A). Uptake of apoptotic red fluorescent labeled material added to cocultures of GFP-microglia and unstained neurons served as a positive control for the overall phagocytic capacity of microglia (Fig. 4B). While only few neuronal membranes were detected in microglia cocultured with normal neurons, the majority of microglia showed uptake of red fluorescent neuronal membranes after desialylation of the neuronal glycocalyx (Fig. $4 A$ ). Similarly, confocal microscopy demonstrated that the uptake of neuronal membranes was increased $(p=4.8 \times$ $10^{-5}$ ) from $72.44 \pm 5.91 \%$ to $113.36 \pm$ $4.50 \%$ after removal of sialic acid from the neuronal glycocalyx (Fig. 4B).

Next, we analyzed whether CR3 is also involved in the microglial uptake of neuronal membranes. Indeed, increased uptake of neuronal membranes due to desialylation was reduced $(p=7.27 \times$ $10^{-6}$ ) after blocking CD11b (69.03 \pm $7.13 \%)$, while control antibody-treated microglia efficiently ingested desialylated neuronal membranes (119.47 $\pm 4.25 \%$; Fig. 4C).

\section{Discussion}

Microglia participate in tissue homeostasis, innate and adaptive immunity (Hanisch and Kettenmann, 2007; Ransohoff and Perry, 2009). In response to injury, ischemia and inflammatory stimuli microglia change from an immunologically silent to an immuncompetent active state. They can migrate to the site of lesion, secrete a wide range of soluble factors and phagocytose cellular debris (Hanisch and Kettenmann, 2007; Ransohoff and Perry, 2009). Apoptotic cells are cleared by microglia via silent phagocytosis, a beneficial process without any signs of inflammation. Recently we demonstrated that TREM2 and its adaptor molecule DAP12 facilitates clearance of apoptotic neurons by microglia without any signs of inflammation (Takahashi et al., 2005). Another potential microglial DAP12-signaling receptor is CR3 (Wakselman et al., 2008). The latter plays an important role in the clearance of structures opsonized with C3bi, a fragment of C3. We now show that microglia via CR3 are capable of clearing desialylated neurites. This process is not accompanied by a change in gene transcription of proinflammatory cytokines (IL-1 $\beta$, TNF $\alpha$ ) or anti-inflammatory cytokine (IL-10) in the coculture system (data not shown). Although CD18, as heterodimeric component of CR3, has not been formally proven to directly bind DAP12, it has been demonstrated to signal via the ITAM of DAP12 (Ivashkiv, 2009) leading to syk kinase activation, which is essential for

A

B

C
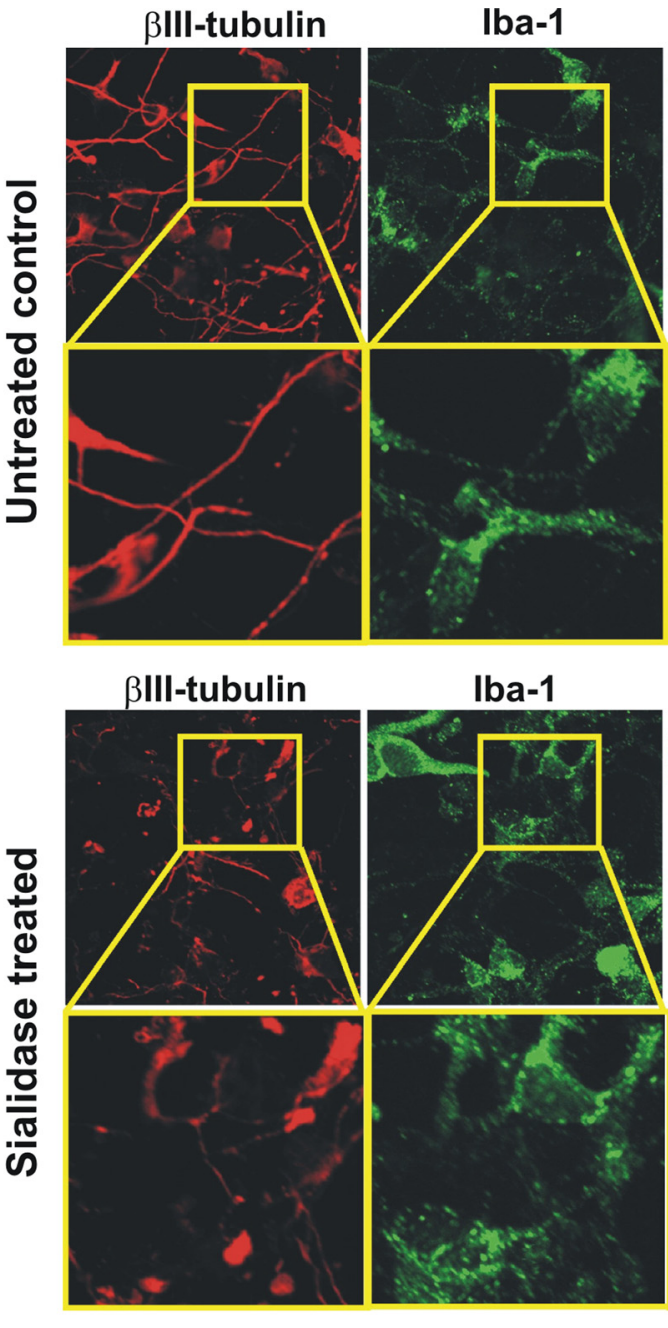

Iba-1
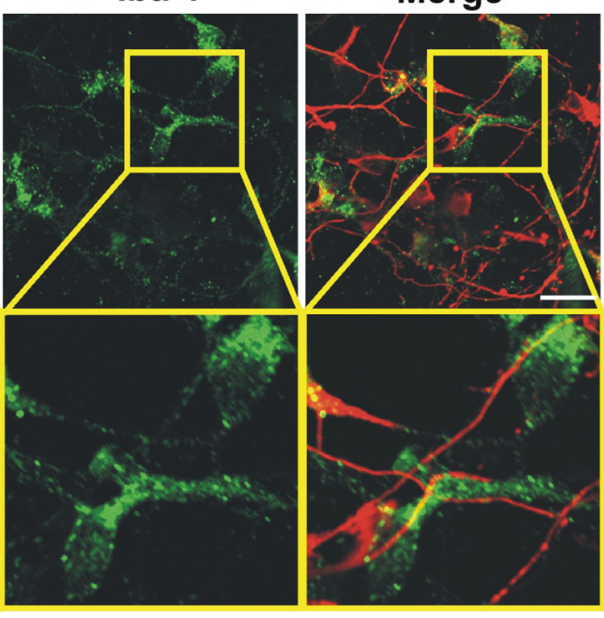

Merge
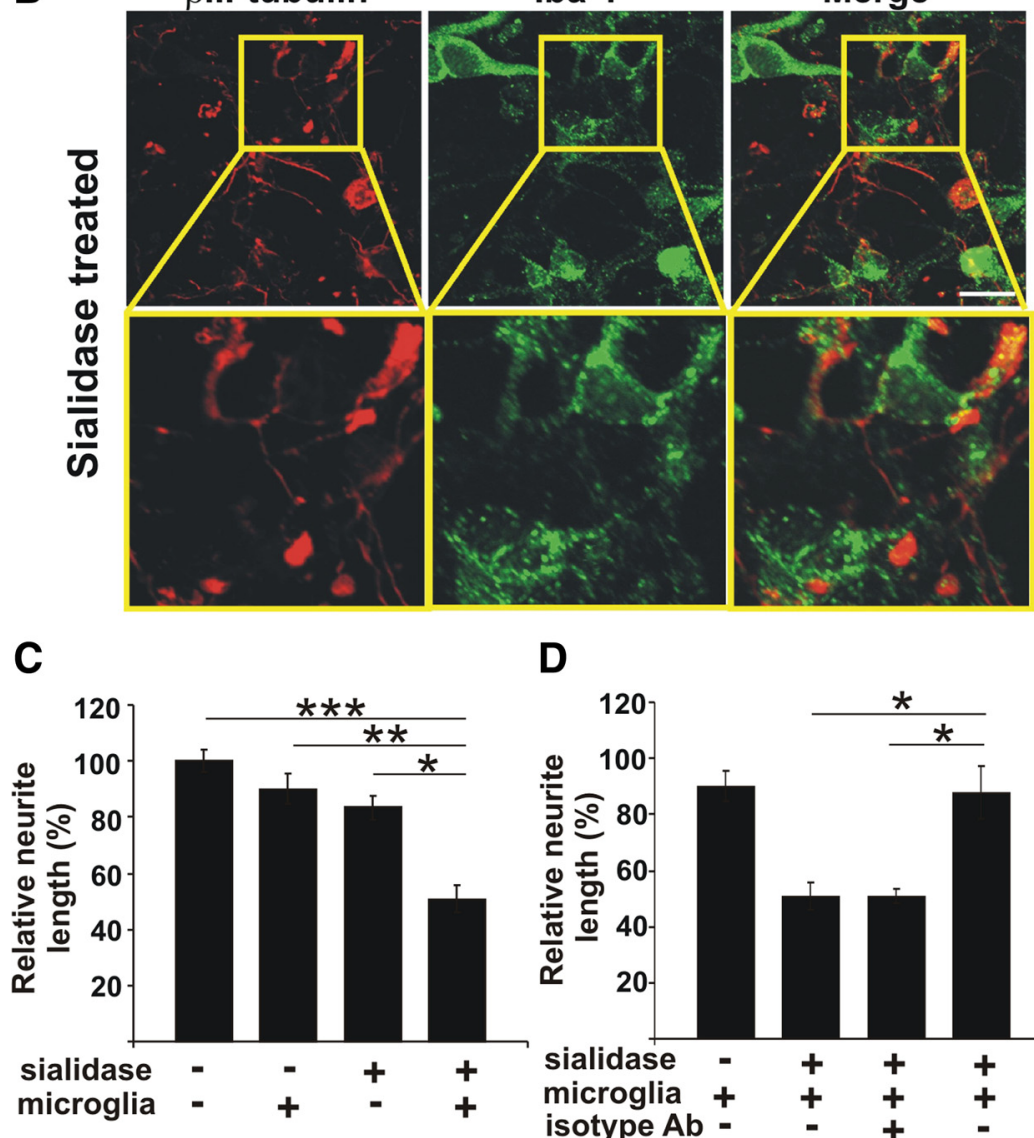

D

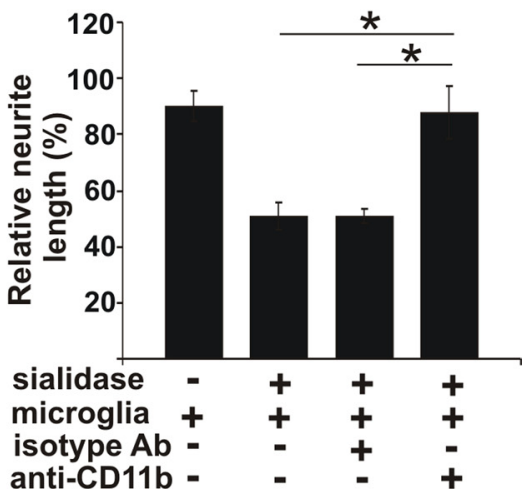

Figure 3. Complement receptor-3-dependent removal of desialylated neurites. $\boldsymbol{A}, \boldsymbol{B}$, Cultured neurons were untreated or sialidase treated and then cocultured for $24 \mathrm{~h}$ with microglia. Cells were immunostained with antibodies directed against $\beta$ IIItubulin and Iba-1. Sialidase-treated neurons displayed shorter and discontinuous neurites compared with untreated neurons. Scale bar, $20 \mu \mathrm{m}, n=3$. C, D, Normal or desialylated neurons (+ sialidase) were cocultured for $24 \mathrm{~h}$ with microglia (+ microglia) and neurite length of neurons was determined. $C$, Addition of microglia reduced the length of desialylated neurites $\left({ }^{* * *} p=0.001\right.$ vs untreated, ${ }^{*} p=0.044$ vs treated, ${ }^{* *} p=0.009$ vs + microglia). $\boldsymbol{D}$, Reduction in desialylated neurite length by microglia was reversed after blockade of CR3 with a CD11b-specific antibody ( + anti-CD11b, ${ }^{*} p=0.016$ ) compared with an isotype control (+ isotype Ab). ${ }^{*}$ vs + sialidase + microglia and vs + sialidase + microglia + isotype Ab, $n=3$.

phagocytosis of pathogens by CR3 (Shi et al., 2006). Thus, CR3-dependent microglial removal of neuronal structures observed in our experiments might be mediated via a DAP12ITAM-syk signaling cascade. Since blockade of CD11b only partially 
A $\quad \begin{gathered}\text { Neuronal } \\ \text { membranes }\end{gathered}$
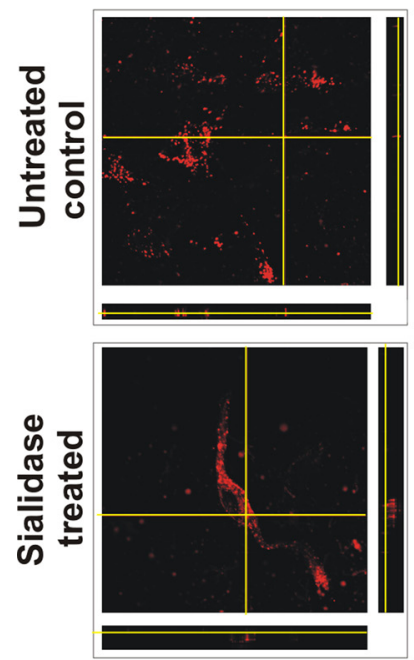

B

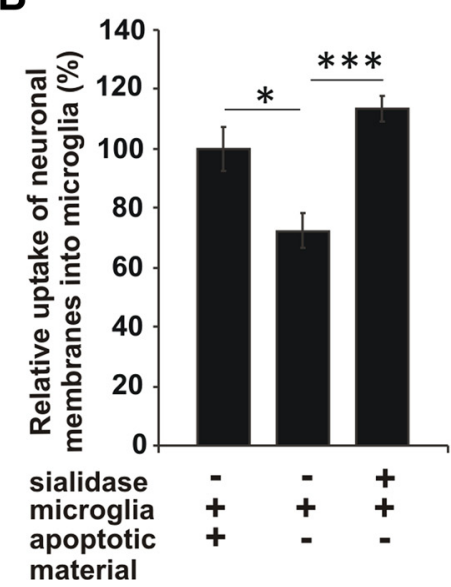

GFP+ microglia
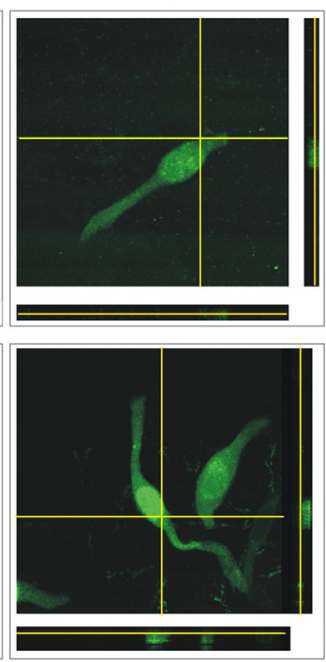

C

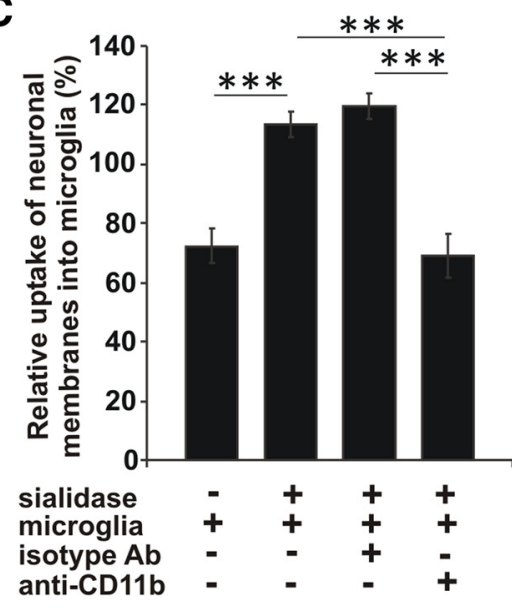

Figure 4. Uptake of desialylated neurons by microglia. Microglia transduced with GFP (GFP + microglia) were cocultured with normal (untreated control) or sialidase-treated neurons, which were labeled with a red fluorescent membrane dye. Uptake of neuronal membranes into microglia was visualized by confocal microscopy and 3D-reconstruction. Uptake of apoptotic red fluorescent labeled material added to the coculture system served as positive control. $A$, After coculture of microglia with desialylated neurons fluorescent-marked neuronal membranes were detected inside the microglia. Scale bars, $20 \mu \mathrm{m}, n=3$. B, C, Quantification of uptake. Few neuronal membranes were detected in microglia cocultured with normal neurons (untreated). Uptake of red fluorescent neuronal membranes into microglia was increased $\left(* * * p=4.8 \times 10^{-5}\right)$ after desialylation of the neuronal glycocalyx (sialidase treated). Few uptake of neuronal membranes was observed after blocking CD11b ${ }^{* * *} p=7.27 \times 10^{-6}$ vs + sialidase, ${ }^{* * *} p=1.9 \times 10^{-7}$ vs + sialidase + isotype Ab). $n=3$.

prevented phagocytic clearance of neuronal structures, other phagocytic receptors might act in concert.

CR3 of microglia does not directly recognize neuronal structures, but we now show that microglia are capable to produced the complement components $\mathrm{C} 1 \mathrm{q}, \mathrm{C} 1 \mathrm{r}, \mathrm{C} 1 \mathrm{~s}$ and $\mathrm{C} 3$ that connect the CR3 to the target structure. This is in line with previous studies demonstrating in vitro the expression of the complement components $\mathrm{C} 1 \mathrm{q}$ and $\mathrm{C} 3$ by unstimulated microglia (Walker et al., 1995) and in vivo a constitutive expression of $\mathrm{Clq}$ in the murine brain (Depboylu et al., 2011). Furthermore, we demonstrate that $\mathrm{Clq}$ is binding to desialylated neurites. Recently, it has been suggested by in vitro studies that $\mathrm{Clq}$ is one responsible factor for microglial removal of neuronal structures (Fraser et al., 2009). While the uptake of apoptotic neurons by microglia was enhanced in the presence of human serum, it was decreased after incubation in C1q-depleted serum (Fraser et al., 2009). Moreover, C1q has been demonstrated to be neuroprotective by improv- ing neuronal viability and neurite outgrowth (Benoit and Tenner, 2011). Treatment of cultured neurons with Clq increased the expression of genes involved in neurite outgrowth (Benoit and Tenner, 2011).

Recent in vivo data demonstrated that the classical complement system was also involved in removal of unwanted synapses that have been tagged by complement for elimination during development (Stevens et al., 2007). C1q and C3 have been shown to be opsonized on certain synapses throughout postnatal CNS development. Mice deficient in C1q or C3 exhibited large sustained defects in CNS synapse elimination, as shown by the failure of anatomical refinement of retinogeniculate connections and the retention of excess retinal innervation by lateral geniculate neurons (Stevens et al., 2007). In a mouse model of glaucoma, Clq localized to synapses in the adult retina early in the disease process (Stevens et al., 2007). In another study, mice with a deficiency of C1q were protected from glaucomainduced neurodegeneration (Howell et al., 2011).

All together data suggest that the first part of the classical complement cascade from $\mathrm{C} 1$ to $\mathrm{C} 3$ is critical for the pruning and elimination of synapses. Our data now shed light on the mechanism of $\mathrm{Clq}$ binding to neuronal cell membranes. We identified sialic acid, a regular membrane component forming the cap of the glycocalyx, as a crucial structure preventing Clq binding. We have not identified the exact binding partner of $\mathrm{Clq}$ on the neuronal membrane, but our data clearly show that desialylated neuronal structures are marked by complement and subsequently cleared by microglia involving CR3. Thus, C1q might bind to oligosaccharide or protein backbones uncovered after removal of the sialic acid cap, inducing $\mathrm{C} 1$ activation and cleavage of $\mathrm{C} 3$ to generate the CR3 binding C3b/iC3b fragment. Desialylation of pentraxin-3 (PTX3), a widely expressed cell membrane anchored protein, enhanced the $\mathrm{Clq}$ binding and the activation of the classical complement pathway (Inforzato et al., 2006). In addition, complement factor $\mathrm{H}$, a soluble negative regulator of the alternative complement pathway, binds $\mathrm{C} 3 \mathrm{~b}$ and polyanionic structures like sialic acid (Ferreira et al., 2010). Moreover, by binding to those structures of the host cells, complement factor $\mathrm{H}$ is involved in inhibiting complement activation through its capacity to increase the decay of the alternative pathway $\mathrm{C} 3$ convertase (Ferreira et al., 2010). Thus, loss of sialic acids might also contribute to enhanced and uncontrolled cleavage of $\mathrm{C} 3$, leading to increased ingestion through CR3.

Our data gives the terminal sugar, namely the sialic acid, an important triggering function. Sialic acids on the glycocalyx are highly regulated. They are hydrolyzed by acidosis and cleaved by sialidases, like the membrane-associated sialidase Neu3 that has 
been detected in the brain (Monti et al., 2000). Moreover, during several pathological conditions like inflammation and cancer oxidative stress arises or is enhanced. The glycocalyx can display different kinds of modifications after being exposed to oxidative stress such as the site-specific degradation of $N$-linked oligosaccharide at $N$-acetylglucosamine residues (Eguchi et al., 2002). During inflammatory processes serum sialic acid concentrations are elevated (Goswami et al., 2003), suggesting that the sialic acid cap of oligosaccharides is removed in pathological conditions involving oxidative stress.

In summary, our data link an altered neuronal glycocalyx to complement binding and subsequent phagocytic removal by microglia. Intact glycosylation protects neurons against the opsonin C1q, prevents classical complement pathway activation and uptake by microglia via CR3.

\section{References}

Benoit ME, Tenner AJ (2011) Complement protein C1q-mediated neuroprotection is correlated with regulation of neuronal gene and microRNA expression. J Neurosci 31:3459-3469.

Beutner C, Roy K, Linnartz B, Napoli I, Neumann H (2010) Generation of microglial cells from mouse embryonic stem cells. Nat Protoc 5: 1481-1494.

Depboylu C, Schäfer MK, Arias-Carrión O, Oertel WH, Weihe E, Höglinger GU (2011) Possible involvement of complement factor C1q in the clearance of extracellular neuromelanin from the substantia nigra in Parkinson disease. J Neuropathol Exp Neurol 70:125-132.

Eguchi H, Ikeda Y, Koyota S, Honke K, Suzuki K, Gutteridge JM, Taniguchi N (2002) Oxidative damage due to copper ion and hydrogen peroxide induces GlcNAc-specific cleavage of an Asn-linked oligosaccharide. J Biochem 131:477-484.

Ferreira VP, Pangburn MK, Cortés C (2010) Complement control protein factor $\mathrm{H}$ : the good, the bad, and the inadequate. Mol Immunol 47:2187-2197.

Fraser DA, Tenner AJ (2008) Directing an appropriate immune response: the role of defense collagens and other soluble pattern recognition molecules. Curr Drug Targets 9:113-122.

Fraser DA, Pisalyaput K, Tenner AJ (2009) C1q enhances microglial clearance of apoptotic neurons and neuronal blebs, and modulates subsequent inflammatory cytokine production. J Neurochem 112:733-743.

Gorlovoy P, Larionov S, Pham TT, Neumann H (2009) Accumulation of tau induced in neurites by microglial proinflammatory mediators. FASEB J 23:2502-2513.

Goswami K, Nandakumar DN, Koner BC, Bobby Z, Sen SK (2003) Oxidative changes and desialylation of serum proteins in hyperthyroidism. Clin Chim Acta 337:163-168.

Hanisch UK, Kettenmann H (2007) Microglia: active sensor and versatile effector cells in the normal and pathologic brain. Nat Neurosci 10:1387-1394.

Howell GR, Macalinao DG, Sousa GL, Walden M, Soto I, Kneeland SC, Barbay JM, King BL, Marchant JK, Hibbs M, Stevens B, Barres BA, Clark AF, Libby RT, John SW (2011) Molecular clustering identifies complement and endothelin induction as early events in a mouse model of glaucoma. J Clin Invest 121:1429-1444.
Inforzato A, Peri G, Doni A, Garlanda C, Mantovani A, Bastone A, Carpentieri A, Amoresano A, Pucci P, Roos A, Daha MR, Vincenti S, Gallo G, Carminati P, De Santis R, Salvatori G (2006) Structure and function of the long pentraxin PTX3 glycosidic moiety: fine-tuning of the interaction with C1q and complement activation. Biochemistry 45:11540-11551.

Ivashkiv LB (2009) Cross-regulation of signaling by ITAM-associated receptors. Nat Immunol 10:340-347.

Khatua B, Ghoshal A, Bhattacharya K, Mandal C, Saha B, Crocker PR, Mandal C (2010) Sialic acids acquired by Pseudomonas aeruginosa are involved in reduced complement deposition and siglec mediated host-cell recognition. FEBS Lett 584:555-561.

Lund J, Takahashi N, Pound JD, Goodall M, Jefferis R (1996) Multiple interactions of IgG with its core oligosaccharide can modulate recognition by complement and human Fc gamma receptor I and influence the synthesis of its oligosaccharide chains. J Immunol 157:4963-4969.

Monti E, Bassi MT, Papini N, Riboni M, Manzoni M, Venerando B, Croci G, Preti A, Ballabio A, Tettamanti G, Borsani G (2000) Identification and expression of NEU3, a novel human sialidase associated to the plasma membrane. Biochem J 349:343-351.

Napoli I, Kierdorf K, Neumann H (2009) Microglial precursors derived from mouse embryonic stem cells. Glia 57:1660-1671.

Neumann H, Kotter MR, Franklin RJ (2009) Debris clearance by microglia: an essential link between degeneration and regeneration. Brain 132:288-295.

Paolicelli RC, Bolasco G, Pagani F, Maggi L, Scianni M, Panzanelli P, Giustetto M, Ferreira TA, Guiducci E, Dumas L, Ragozzino D, Gross CT (2011) Synaptic pruning by microglia is necessary for normal brain development. Science 333:1456-1458.

Ransohoff RM, Cardona AE (2010) The myeloid cells of the central nervous system parenchyma. Nature 468:253-262.

Ransohoff RM, Perry VH (2009) Microglial physiology: unique stimuli, specialized responses. Annu Rev Immunol 27:119-145.

Shi Y, Tohyama Y, Kadono T, He J, Miah SM, Hazama R, Tanaka C, Tohyama $\mathrm{K}$, Yamamura H (2006) Protein-tyrosine kinase Syk is required for pathogen engulfment in complement-mediated phagocytosis. Blood 107:4554-4562.

Stevens B, Allen NJ, Vazquez LE, Howell GR, Christopherson KS, Nouri N, Micheva KD, Mehalow AK, Huberman AD, Stafford B, Sher A, Litke AM, Lambris JD, Smith SJ, John SW, Barres BA (2007) The classical complement cascade mediates CNS synapse elimination. Cell 131:1164-1178.

Takahashi K, Rochford CD, Neumann H (2005) Clearance of apoptotic neurons without inflammation by microglial triggering receptor expressed on myeloid cells-2. J Exp Med 201:647-657.

Tateno H, Crocker PR, Paulson JC (2005) Mouse Siglec-F and human Siglec- 8 are functionally convergent paralogs that are selectively expressed on eosinophils and recognize $6^{\prime}$-sulfo-sialyl Lewis $\mathrm{X}$ as a preferred glycan ligand. Glycobiology 15:1125-1135.

Wakselman S, Béchade C, Roumier A, Bernard D, Triller A, Bessis A (2008) Developmental neuronal death in hippocampus requires the microglial CD11b integrin and DAP12 immunoreceptor. J Neurosci 28:8138-8143.

Walker DG, Kim SU, McGeer PL (1995) Complement and cytokine gene expression in cultured microglial derived from postmortem human brains. J Neurosci Res 40:478-493.

Ziegenfuss JS, Biswas R, Avery MA, Hong K, Sheehan AE, Yeung YG, Stanley ER, Freeman MR (2008) Draper-dependent glial phagocytic activity is mediated by Src and Syk family kinase signalling. Nature 453:935-939. 